\title{
APPLICATION OF CLOUD SERVICES IN EDUCATION
}

\author{
G. Kiryakova* \\ Faculty of Economics, Trakia University, Stara Zagora, Bulgaria
}

\begin{abstract}
Educational institutions in information society rely heavily on information and communication technologies. They allow them to follow innovative pedagogical paradigms and approaches and implement modern forms of training that are tailored to the needs and characteristics of the new generation of learners. More and more educational institutions are turning to the use of cloud services, because they are extremely effective alternative for providing the high quality resources and services to all participants in the learning process at an affordable price.

The main objective of this study is to present the most popular cloud services - cloud-based office suites and cloud storage services, focusing on their use in education.
\end{abstract}

Keywords: Cloud computing, education, cloud-based office suites, cloud storage services

\section{INTRODUCTION}

Modern education is invariably linked to the widespread use of information and communication technologies (ICT). They allow realizing training which is based on new pedagogical paradigms where the learner is central figure and actively participates in the learning process, interacts and collaborates with all other participants.

For many schools cloud computing model offers efficient, in terms of expenses, solution to the problem of how to provide resources and services to the rapidly growing number of students without making significant capital investments in hardware and software that also have to be maintained (1). On the other hand, the continuous access to information and the options for sharing it are among the most significant advantages of cloud computing. Currently, cloud-based services are very important and necessary for modern education.

\section{NATURE OF CLOUD COMPUTING AND MAIN FEATURES}

Cloud computing is not another technological phenomenon. It is based on a number of existing technologies. Cloud computing is the result of the evolution of different technologies that come together and change the way organizations build their IT infrastructure and host their systems (2).

\footnotetext{
*Correspondence to: Gabriela Kiryakova, Faculty of Economics, Trakia University, Bulgaria, gabriela@uni-sz.bg
}

According to (3) cloud computing is "a model for enabling ubiquitous, convenient, ondemand network access to a shared pool of configurable computing resources that can be rapidly provisioned and released with minimal management effort or service provider interaction".

The main characteristics of cloud computing that distinguish them from other technologies are $(3,4)$ :

- On-demand self-service. Users can unilaterally request and receive computing resources when they need them.

- Permanent network access. Computing resources are available anytime from anywhere in the world over the network through standard mechanisms. Users can use various devices and platforms that facilitate access to services.

- Pooling and sharing of resources. Computing resources are pooled and provided to many users. They are not set aside and fixed between the consumers in advance. The resources are dynamically assigned according to users' needs.

- Rapid elasticity. Users can dynamically change (increase or decrease) the rented resources depending on their current needs. The possibility of scaling the rented infrastructure allows users to react promptly to dynamically changing needs.

- Pay-per-use. Cloud computing is characterized by payment for only actually used computing resources, therefore the price depends on the level of users activity. 
CLOUD COMPUTING IN EDUCATION

Traditional forms of e-learning require significant investments for building appropriate IT infrastructure and regular costs for maintaining, upgrading and managing hardware and software. The increased costs for hardware and software are beyond the strength of the majority of schools that have limited financial resources. Cloud computing is one of the global trends in ICT development and its implementation in education can be an effective way to solve existing problems and achieve goals at minimal cost.

\section{ADVANTAGES OF CLOUD COMPUTING FOR EDUCATION}

Cloud computing offers an infrastructure, platform and educational services that create an affordable and innovative learning environment. Such an environment allows collaboration between all participants in the learning process and between different educational institutions that reflects on the quality of education.

The benefits of the cloud computing model are for teachers and students as well as for the educational institutions.

The advantages of cloud services for education can be considered from different aspects (4-7). The use of applications and services, delivered by external providers, is a cost-effective and efficient solution for educational institutions and allows enhancing financial flexibility. The advantages of cloud computing model, compared to the traditional approach, are expressed in: reduced costs for hardware and software as well as reduced costs for IT staff; payment for actual consumption; provision of many free services. Cloud computing offers a faster return of investments and dealing with rapidly changing software and hardware needs at a lower cost. There is a flexibility of the employment of resources combined with economic efficiency.

The educational institutions can implement effectively their strategy through cloud computing without the need to take care of its physical (hardware and software) provision. They have options to acquire and implement new IT solutions and to hire IT resources quickly. Service providers ensure the maintenance and management of IT resources. Cloud computing guarantees the use of modern ICT by educational organizations, something that cannot be achieved if they use their own IT infrastructure.

Cloud computing provides an easy and unrestricted access to services and resources at any time and place through a variety of devices both for teachers and students. The comprehensive accessibility by different devices enables the realization of ideas for mobile and lifelong learning - mobile learning that is expressed not only in using mobile devices, but primarily in the mobility of participants in the learning process.

\section{CLOUD SERVICES WITH} APPLICATIONS IN EDUCATION

Among the most popular cloud services that are successfully implemented in education are cloud-based office suites and storage services (cloud storage).

\section{CLOUD-BASED OFFICE SUITES}

Cloud-based office suites, also known as online office suites or cloud-based collaboration tools in real time, are office suites that are provided as SaaS services.

Among the most popular cloud office suites, used in education, are Google Apps for Work, Office 365, Zoho Office Suite, Apple Productivity Apps, Amazon WorkDocs, Thinkfree Online, Live Documents.

Cloud-based office suites are available through Web browser, so they are platform and hardware independent. There is no need to install and configure software on local computers that allows students and teachers to work with cloud-based office suites on a variety of devices, including mobile. Using cloud-based office suites the idea of BYOD (Bring Your Own Device) can be implemented.

Online office suites offer opportunities for sharing and collaboration, because data are stored in the cloud, allowing access to multiple users from different locations through a variety of devices (8). Via cloud-based office suites teachers and students can work together on shared documents, projects and tasks in real time. Everyone can see and comment other participants' activities, which increases the productivity of collaboration. Online packages are a tool for creating interactive learning environment where students participate actively in learning activities, collaborate, interact and communicate with all participants in training. For educational organizations this is an opportunity to implement the ideas of active learning and social constructivism paradigm. Cloud office suites are an appropriate tool for collaboration between students on common projects and tasks - each student performs his individual tasks in a group project, and the results are summarized in a joint document. Students can "assess" and 
edit their colleagues' assignments or project works during collaboration, which leads to development of critical thinking, skills for analysis and evaluation, motivate own opinion, etc. Collaboration encourages learners to participate actively in performing different tasks, because their actions and results are visible to others. Teachers can assess learners' knowledge and skills objectively, since there are options to trace and establish the personal contribution of each student.

Cloud office suites provide opportunities for working offline and subsequent synchronization (when an Internet connection is available), which gives teachers and students the freedom to work anytime, anywhere and at their own pace, eliminating dependence on physical location and time differences.

The base versions of cloud siutes are usually free and for versions with additional features are available different plans. Cloud siutes are offered free or with big discounts for educational institutions that makes them even more attractive.

The main components of cloud-based office suites are:
KIRYAKOVA G.

- Applications for creating and editing documents - word processing, spreadsheets and presentations. Some packages include applications for creating and editing graphical objects, database management systems, software for publishing content (applications for creating Web sites without requiring Web programming skills, content management system, Blog, Wiki, etc.). Availability of facilities for storing documents as templates is a premise for creating libraries (repositories) with reusable learning materials and accelerating and facilitating the process of developing new learning content.

File sharing is one of the main advantages of cloud office suites as a prerequisite for effective real-time collaboration between multiple users - Figure 1. Users can view and edit shared files simultaneously and changes are visible to all active participants (they are displayed on the screen) - Figure 2 . Opportunities for comments and notices enhance collaboration and can be used for sharing ideas, discussions and communication. A history of changes is available and users can track changes and revert to older versions.
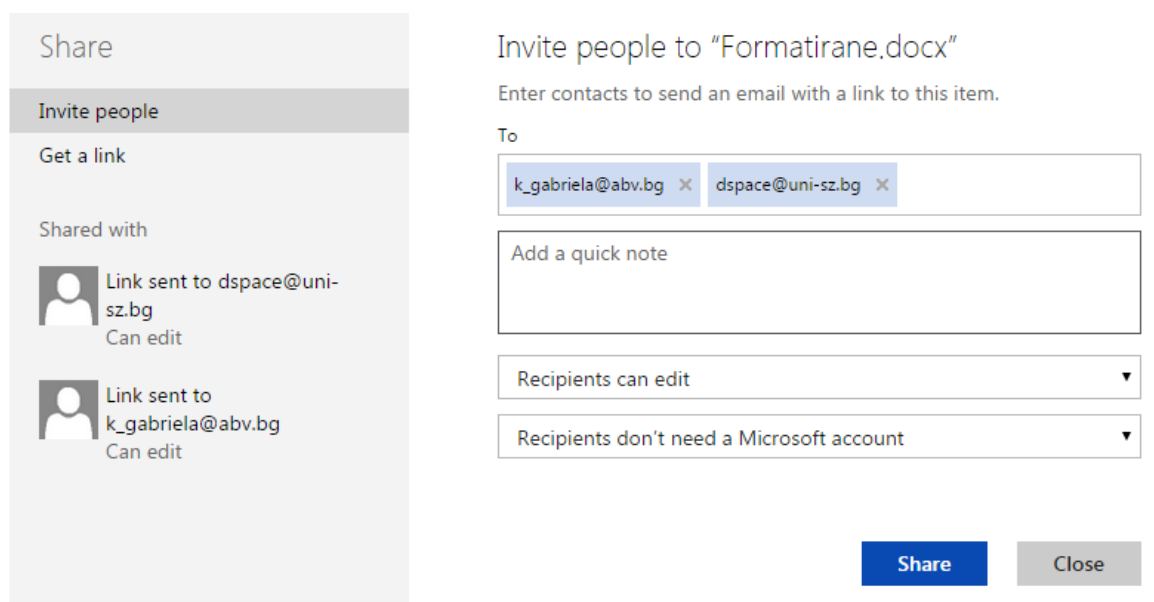

Figure 1. Sharing files in Office Online (Office 365).

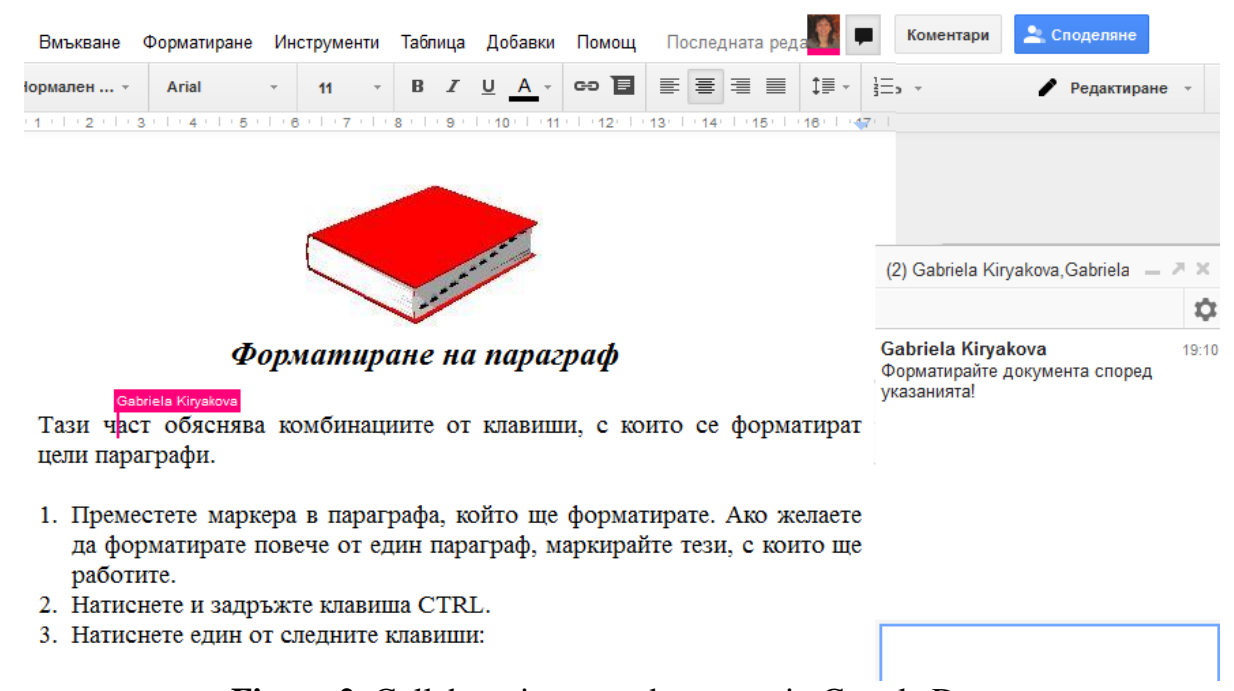

Figure 2. Collaboration on a document in Google Docs. 
Some cloud packages offer additional functionality to collaboration. Zoho Docs provides options for assigning and tracking tasks to individuals on joint projects. Teachers can assign individual tasks to learners, monitor their work and distinguish the contribution of each participant.

- Applications for communication - e-mail, instant messaging (IM), online conferences, forums, and others. They enrich the possibilities for communication (synchronous and asynchronous) between participants in the learning process and offer new and more engaging forms for active connection in real time. Options for adding comments to shared documents allow students to share ideas, seek help or advice in difficult tasks.

- Calendars and tools for contacts management. Users can manage upcoming and ongoing events and meetings via calendars. Personal calendars enhance management of users' individual tasks. Shared calendars can support learning process and all activities taking place in educational institutions. They can be used for planning and notification ьфдкш different events and creating weekly, monthly or annual classes' schedules. Shared calendars may help students organize collaborative work on group projects more effectively. On the other hand, teachers can use and share calendars with their colleagues to define schedules of meetings and events, educational activities and tasks with deadlines and support their timely execution. Some calendars (Microsoft 365) offer options for assigning tasks. Teachers can create and assign tasks to students and involve them to participate actively in both the academic and social life of the group.

- Storage space. Documents, spreadsheets and presentations, created by cloud-based suites, are stored in a centralized repository in the cloud. Users have an access to the repository and updated versions of all documents. Most of cloud office suites offer limited free storage space and paid versions allow its extention. Storage space services provide tools for managing files by organizing them into folders, adding file descriptions, allowing usage of different criteria in searching through the collections of stored documents.

- Specific educational applications. Some vendors, such as Microsoft and Google, add special educational applications to their office suites, making them extremely popular among educational institutions.
Classroom is an educational application, part of Google Apps, which allows teachers to create, assign and collect students' individual assignments (9). Teachers create courses (classes) and make assignments for individual or group work. They can add files from local computers, Google Drive, YouTube or a link to any Web page, since via Classroom there is integration between Google Docs, Google Drive and Gmail. Teachers can view tasks during the process of their performance, make comments, send feedback with recommendations or reviews to help students and evaluate them. Students can join classes, execute their assignments, communicate with teachers, comment and discuss with other students. Learners work on assignments directly in Google Docs and can add files from local computers, Google Drive or a link to a Web page or can create new Google Drive files of different types (documents, spreadsheets, presentations).

OneNote Class Notebooks (Microsoft) is a tool for planning and performing lectures, tutorials, exercises, assignments and quizzes. Teachers are able to create interactive lessons that include various content - text, graphics, links to Web sites or pages from the notebook, tables, audio and video materials (10). The application provides a personalized workspace for each student - section Student Notebooks. In section Collaboration Space students and teachers can work together on projects and shared tasks. Students have opportunities not only to view the available content, but also to add new or edit existing materials. Section Content Library is also for publishing educational materials, which are available for students, but only for viewing or downloading without editing capabilities.

Sway (Microsoft) is an application that facilitates teachers in creating and sharing interactive tutorials, educational resources and documents with different content. Sway offers interactive Web-based canvas, where various elements (text, graphics, videos, diagrams and others) can be added and combined to create a whole product. Teachers can visualize educational materials and make them more attractive or create collections with students' works and show their results and progress. Learners can use Sway as a tool to present their creative ideas on projects or assignments. Created canvas can be shared with other users by links and are accessible via Web browsers.

Zoho Wiki is a tool for creating wikis. Although the application is not designed for educational institutions, it can be used by them as a tool for collaboration between all 
participants in the learning process. The result of collaboration is generation of a new content and knowledge. Wiki pages are created using a WYSIWYG editor and include various elements - text, graphics, dynamic content like videos, presentations and other (11).

In conclusion, it can be summarized that cloudbased office suites provide basic functionalities of office suites - creating text documents, spreadsheets and presentations. The main advantage of cloud packages is the ability for sharing files and collaboration that increases productivity. Cloud offices include tools to manage contacts, communication (synchronous and asynchronous) and calendars. Files are available through a variety of devices (including mobile) and the possibilities for data synchronization between multiple devices and users are an essential characteristic of cloud suites. Some of the packages include specific educational apps.

\section{CLOUD STORAGE}

Cloud Storage is a model for storing data on multiple virtual servers that users can access from anywhere and anytime using multiple devices (8).

Storing data in the cloud is gaining popularity because of the potential for collaboration between multiple users, which increases the productivity of work.

Among the most popular cloud storage services are Dropbox, Box, Google Drive, One Drive, iCloud Drive and others.

The offered free disk space per user varies in different services. Free disk space in Dropbox is 2,5GB and can be increased up to 16GB by attracting users to Dropbox. Box offers 10GB free disk space, which can be increased to 100GB in paid versions. Each personal account in Google Drive has 15GB free disk space in the cloud. Users of Google Apps for Education have 30GB of disk space, which is shared between Gmail, Drive and Google+ photos. The available space can be extended by purchasing additional. One Drive users have $5 \mathrm{~GB}$ of storage for free and they can purchase plans to expand their storage limit. iCloud Drive offers 5 GB free disk space, which is shared by iCloud Backup, iCloud Drive, iCloud Photo Library, iCloud Mail and iCloud applications.

Cloud storage services have a lot of advantages (12):

- Accessibility. The main advantage of cloud storage is the ability to access stored files anytime and anywhere via different devices.
Cloud storage services allow teachers to organize all training materials in one place and make them available both for their colleagues and for students. Learners have an access to educational materials anytime, anywhere, via any device they own. They have the opportunity to learn and work together with other students, following their own pace and preferences. Cloud storage services help realize the ideas of comprehensive education and continuous access to learning resources.

- Synchronize data between different devices and users. Most of the cloud storage services offer opportunities to work offline there is a desktop version of the application that can be installed on local devices and users can work offline. Files are automatically update and synchronize between different devices of the user and between multiple users working with them when an Internet connection is established. Files synchronization between multiple users and their various devices supports collaboration all changes (additions, edits) are automatically available to all participants and they always work with the latest version of the documents.

- Compatibility. More and more educational institutions are turning to the idea of BYOD in order to allow students to use their everyday devices not only for entertainment but also for learning. An essential feature of cloud storage services is to support multiple platforms to ensure interoperability and use of various devices.

- Sharing files. Sharing of stored files is one of the most important characteristics of cloud storage services. A significant part of the applications (Box, Google Drive, One Drive) allow sharing files with external users (who do not have accounts) through a public links. Sharing via links can be with a higher level of protection (a password for access, valid for a certain period of time, is required) or imposed restrictions on downloading files. Another important feature is the different access levels for shared files - read only, options for editing, shared ownership - Figure 3. The ability to share files creates prerequisites for building repositories of educational materials, which can be available to all learners. Sharing resources between teachers contributes to spread and reuse of the learning content. As a result the development of new materials and courses speeds up and their quality improves. On the other hand, students can share their projects and collaborate with other students on group tasks. 


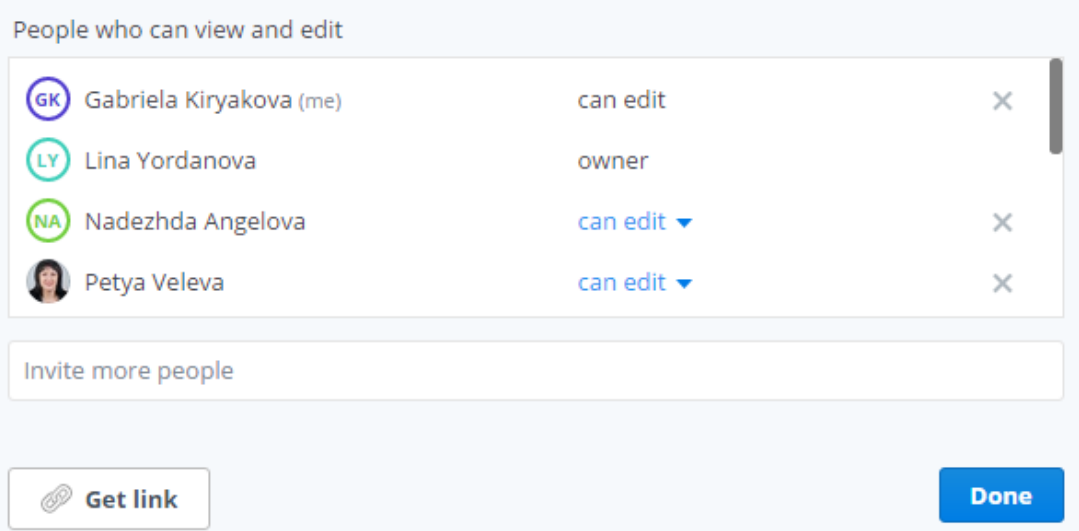

Figure 3. Sharing files in Dropbox.

One of Dropbox innovations is to provide opportunities to collect files from other users file requests (13). There is a two-way transfer of information - sharing (provision) and collecting (receiving) files. Via file requests teachers can easily collect files from students completed assignments, projects, tasks and other materials and evaluate them. They create a folder and send requests to students who upload files into it, even without having Dropbox accounts. File requests greatly facilitates teachers in their scientific and research activities when collecting and summarizing information from many participants takes time and considerable efforts.

- Collaboration. Opportunities for simultaneous access to the latest version of files by many users enables collaboration between them. The collaboration includes communication between participants in different forms - comments, chat and others and allows achieving a higher level of interaction, increasing productivity and improving the quality of work. Most of the storage services provide integration with office applications and offer higher degree of cooperation - Figure 4. In education, storage services are an opportunity for teachers to collaborate with their colleagues on creating learning resources and content as well as on research projects. On the other sibe, teachers have an access to students' assignments and can monitor and track their performance and activities, giving them guidance and timely feedback, and evaluate their contribution to the shared tasks. Cloud storage services offer tools to create a collaborative environment for all learners and to engage them into learning activities.

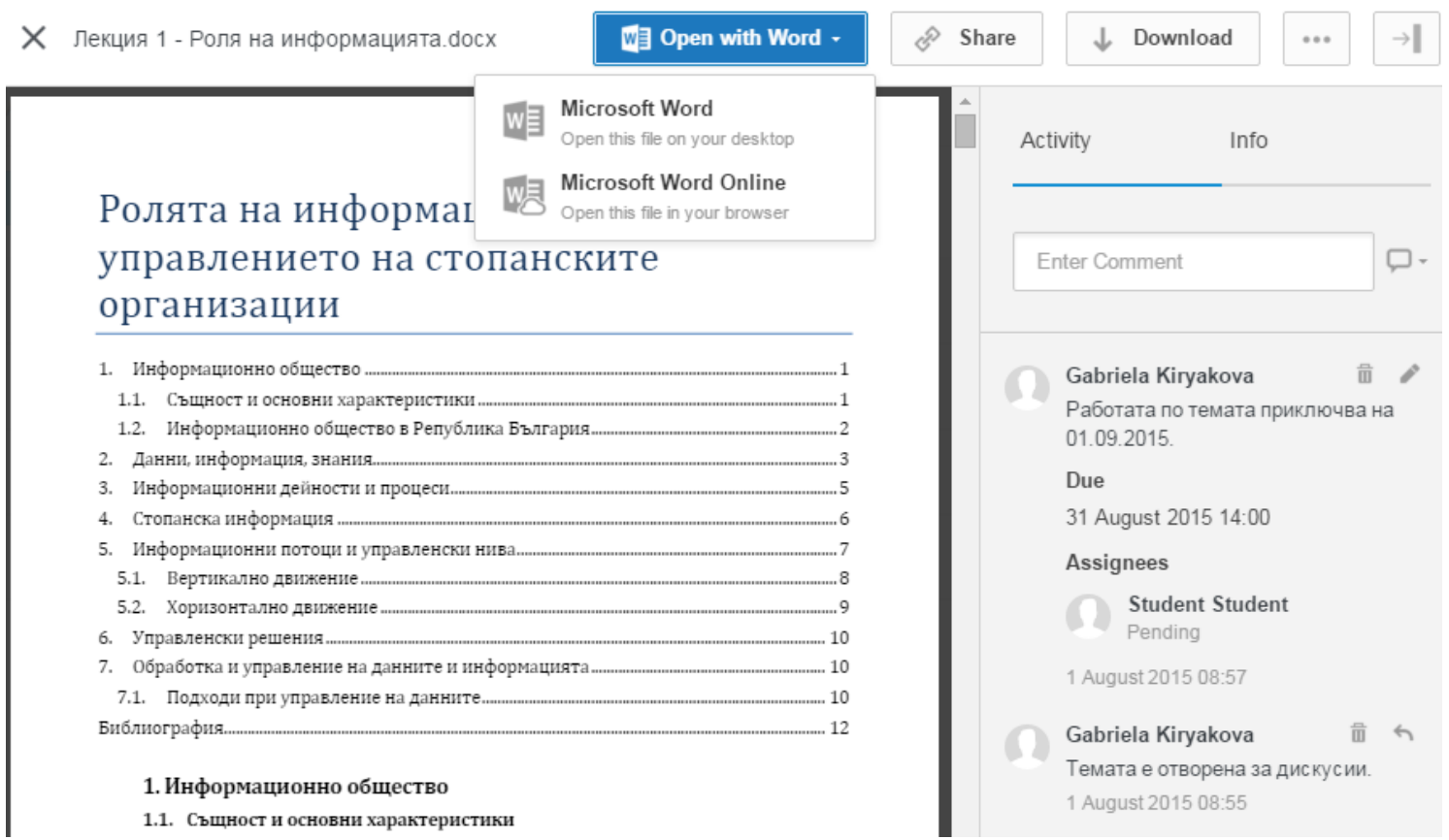

Figure 4. Working with sharing files in Box. 
For example, Google Docs offers various editing modes in collaboration (14). In Editing mode changes that participants make are visible to all in real time. In Suggesting mode changes are considered as proposals that can be accepted or rejected and there are conditions for effective management of collaboration. One Drive maintains history of documents version and allows a return to previous versions. iCloud Drive differs from other cloud storage services regarding to the ability to share files. iCloud Drive provides options for sharing file and applications (calendars, emails) between multiple devices of a user. Files sharing and collaboration between different users are not available features in iCloud Drive.

- Collaboration. Many cloud storage services are integrated with a wide range of applications - office suites, other storage services, social networks and more.

For example, Zoho Docs provides integration with Google Drive - Zoho users can import documents that are stored in Drive. There is synchronization between Dropbox and Google Drive - when users add files to Dropbox, they are automatically added to Google Drive. Existing integration between Google Drive and Facebook and Instagram allows storing images, posted on social networks, in Google Drive.

Dropbox and Box are fully integrated with Microsoft Office applications. Integration includes options for editing Dropbox/Box files through Microsoft Office Online directly in web browsers and access to cloud services directly from Office Online.

There is a full integration of OneDrive with Office Online, as OneDrive is integrated directly into the new versions of Windows (version 8.1 and up).

In conclusion, cloud storage services provide options for storing different types of content, which actual versions (due to synchronization between devices and users) are accessible for users at any time and place through different devices. Sharing data and options for collaboration, accompanied by various means of communication, help achieve greater efficiency and productivity of all learning and administrative activities.

\section{CONCLUSION}

Cloud computing supports the implementation of various forms of electronic, distance and blended learning, which are extremely popular due to the penetration of ICT and have significant advantages over traditional forms of education.

One of the main reasons for transition to a cloud computing model is the fact that traditional forms of e-learning require significant investments - building appropriate IT infrastructure, expenses for its maintenance, need for highly qualified IT staff to operate and manage it. The increased costs and reduced financial resources urge educational institutions to seek solutions to overcome existing constraints and to provide conditions for effective learning process, consistent with the new educational trends and paradigms.

Cloud computing ensures comprehensive access to modern ICT for a wide range of users at any time and place via different devices, which is a prerequisite for the implementation of the ideas of lifelong learning. There is an effective use of available IT equipment with opportunities for renting powerful computing resources when they are needed. Using cloud services, educational institutions can concentrate on their main activities - training and research and fulfill them in the most efficient manner.

Cloud computing is extremely important and necessary for modern education. It transformes the role of ICT in training, supports and accelerates the processes of creating and providing an interactive learning environment where all participants have continuous access to diverse, high-quality educational resources and activities and work collaboratively.

\section{REFERENCES}

1. Al-Zoube, M., E-Learning on the Cloud, Int. Arab J. e-Technol. 1.2, 58-64, 2009.

2. Reese, G., Cloud Application Architectures - Building Applications and Infrastructure in the Cloud, O'Reilly Media, ISBN 978-0596-15636-7, 2009

3. NIST Cloud Computing Standards Roadmap, Special Publication 500-291, Version 2, July 2013, http://www.nist.gov/itl/cloud/upload/NIST_ SP-500-

291_Version2_2013_June18_FINAL.pdf

4. Sclater, N., Cloud computing in education, Policy Brief, UNESCO Institute for Information Technology in Education, 2010

5. Cisco Systems, Cloud 101: Developing a Cloud-Computing Strategy for Higher Education, White Paper, 2012, http://www.cisco.com/c/dam/en/us/services /collateral/services/serviceseducation/cloud_101_higher_education_wp .pdf, 
6. IBM Global Technology Services, Applying the cloud in education An innovative approach to IT, White Paper, http://www-

935.ibm.com/services/be/en/cloudcomputing/cloud_edu_en.pdf

7. Sultan, N., Cloud computing for education: A new dawn?, International Journal of Information Management 30.2, 2010, 109116.

8. Wikipedia, https://www.wikipedia.org/

9. Training Center, Google for Education, https://edutrainingcenter.withgoogle.com/tr aining. http://onenoteineducation.com/

11.Zoho Office Suite, https://www.zoho.com/

12.Catalano, H., Best Cloud Storage Providers for 2016, 2015,

http://www.thesimpledollar.com/bestcloud-storage-provider/

13.Dropbox, https://www.dropbox.com/business

14.Google Apps Administrator Help Center, https://support.google.com/a/answer/13901 $9 ? \mathrm{hl}=\mathrm{en}$ 